\title{
AVALIAÇÃO EXTERNA DE AMBIENTES DE E-COMMERCE
}

\section{Tomé Rodrigues e António Andrade*}

\begin{abstract}
Resumo: Na vasta literatura existente sobre avaliação de websites elou portais, qualidade, web-design e usabilidade são as dimensões mais utilizadas na criação de modelos de avaliação, no entanto, encontramos evidencias para a importância da estratégia como dimensão condicionadora de todas as outras. Não encontrando registo da união das quatro dimensões num único modelo, apresentamos uma proposta de visão integrada, para uma avaliação externa de ambientes de e-commerce, uma vez que a probabilidade de sucesso de um website depende da integração das dimensões referidas e fatores externos capazes de acrescentar valor ao consumidor.
\end{abstract}

Palavras-chave: Avaliação, Website, Portal, Modelo, Qualidade, Usabilidade, Design, Informação, Utilizador, Internet.

Abstract: In the vast literature on Web site evaluation and / or portals, quality, usability and web design are the most used dimensions in the creation of evaluation models, however, we found evidence of the importance of strategy as a dimension that all the others depend on. As we haven't found any record of the union of the four dimensions in a single model, we present a proposal for an integrated vision, for an external evaluation of e-commerce environments, since the probability of success of a website depends on the integration of these dimensions and external factors that can add value to the consumer.

Keywords: Evaluation, Website, Portal, Model, Quality, Usability, Design, Information, User, Internet.

\footnotetext{
* UCP - Universidade Católica Portuguesa, FEG - Faculdade de Economia e Gestão.
} E-mail: aandrade@porto.ucp.pt 


\section{INTRODUÇÃO}

Nos dias de hoje a Internet assume um papel cada vez mais importante nas vidas de pessoas e empresas. É um marketplace global onde os agentes económicos interagem e evoluem.

Sendo os websites a base da Internet estamos, obviamente, muito familiarizados com eles. Sem eles o e-commerce não faria sentido!

Porém, por diversas razões, nem todos os sites ou portais têm o sucesso que os seus investidores desejam. Este facto leva-nos a uma questão: poderá a avaliação externa de ambientes de e-commerce apoiar os investidores e/ou gestores a desenvolver um website com superior potencial de sucesso? Desta questão decorre uma outra: quais as dimensões que devem ser avaliadas tendo em consideração o site/ industria/ target?

No intuito de responder a estas questões procedemos a uma extensa revisão literária, que nos levou a apresentar a nossa visão representada num modelo a ser testado futuramente, que pensamos ser de interesse académico para os investigadores no domínio de avaliação de websites e/ou portais.

\section{WEBSITE}

De acordo com Carvalho et al. (2005) um website corresponde a um conjunto estruturado de páginas web que representam uma entidade ou uma pessoa. Os websites estão acessíveis através de um browser ${ }^{\dagger}$.

Devido à diversidade de formatos da web, esta segundo Lévy (2000), está muito próxima do conceito de unimédia, ou seja, os sites estão em constante construção e renovação podendo ser alterados em qualquer momento pelo webmastert.

\subsection{Funções do Website}

"Um site pode dispor informação ou pode proporcionar atividades pré-definidas, isto é, pode ter funcionalidades que permitam ao utilizador fazer mais do que observar e navegar" (Carvalho et al., 2005:21).

Deste modo um site pode integrar uma ou mais funções que se apresentam seguidamente:

\section{- Expositor de informaçãa;}

\footnotetext{
${ }^{\dagger}$ Explorador da internet, p.e. Firefox, Opera ou Safari

* Profissional que desenvolve a arquitetura de um website
} 


\section{- Coletor de informação;}

- Meio de comunicação entre utilizadores;

- Instrumento de trabalho.

A dinâmica da atividade do utilizador está intrinsecamente relacionada com a sua aprendizagem (Carvalho et al., 2005; Simões, 2005). As interações propostas pelos autores estão organizadas, gradativamente, da seguinte forma:

- Ativa-animação;

- Escreve-e-envia;

- Escreve-e-verifica;

- Manipula-e-verifica;

- Insere-e-verifica (Simões, 2005).

Segundo Kim (2004) as empresas tendem a ser mais eficazes na utilização dos seus sites, como tal, estes tendem a ser cada vez mais complexos. Para algumas organizações os sites funcionam como repositórios de informação para os vários intervenientes e para o público em geral. Para outras organizações, os sites, também, oferecem capacidades de operação, proporcionando um mecanismo adicional para servir melhor os clientes.

\section{PORTAL}

A ideia de um portal é coletar informações de diferentes fontes e criar um único ponto de acesso à informação, funções e/ou serviços que são relevantes para o trabalho de uma pessoa ou para os seus interesses pessoais (Granic et al., 2010).

De acordo com Granic et al. (2010) os portais são diferentes dos websites, pois proporcionam uma mistura de informação, aplicações e serviços, deste modo os portais têm mais do que apenas informações para oferecer, uma vez que são baseados em tecnologias web mais avançadas que vão além da interface simples de páginas web que um website típico apresenta.

Pode dizer-se que um portal é um híbrido aos níveis de web-design e de aplicações devido a uma forte exigência de flexibilidade para os manter interessantes para o público (Blankenship, 2001).

Os Portais podem ser classificados em duas grandes categorias:

- Portais horizontais: cobrindo muitas áreas, são muitas vezes referidos como "megaportais", tendo como alvo toda a comunidade da Internet. Geralmente contêm motores de busca e fornecem capacidade para o utilizador personalizar a página (Granic et al., 2010). 
- Portais verticais: com foco numa área funcional, estes diferem apenas pelos seus objetos mais específicos e conteúdo, oferecendo informações e serviços personalizados para públicos específicos (Granic et al., 2010).

Assim, duas gerações de portais podem ser diferenciadas (Granic et al., 2010):

- Portais de primeira geração que tendem a apresentar uma arquitetura de software monolítico, o que compromete o desenvolvimento e manutenção do portal;

- Portais de segunda geração, que permitem aos utilizadores criar uma ou mais páginas pessoais, composto por páginas personalizáveis (aplicações interativas mini web que permitem fragmentos de marcação por exemplo, notícias, clima, desporto e assim por diante) que o portal pode agregar (Bellas, 2004).

\subsection{Funcionalidades do Portal}

De acordo com Granic et al. (2010) os portais têm três funcionalidades principais:

- Busca e navegação;

- Integração de informações;

- Personalização.

Atualmente os portais horizontais de informação estão inevitavelmente a substituir a imprensa escrita que se apresenta em declínio, assim, o papel do portal de informação tende para se tornar o líder de média informativa (Granic et al., 2010).

\section{QUALIDADE NO E-COMMERCE}

Os casos de sucesso no comércio eletrónico mostram-nos que os principais determinantes do sucesso ou fracasso não são apenas presença na web, ou baixo preço, mas sim a entrega de um site de alta qualidade (Rababah et al., 2011). No mesmo sentido os autores referem que os clientes estão dispostos a pagar mais por um serviço de qualidade, assim sendo, a qualidade assume hoje um papel determinante na atração e retenção de clientes e em consequência o sucesso da empresa na web. Os autores Bai et al. (2008) acrescentam que a qualidade do site é um aspeto crítico na criação de tráfego e fomenta o consumo de produtos online.

Para Liu e Arnett (2000) existem quatro fatores que contribuem para o sucesso de um site: qualidade da informação e serviços; a utilização do sistema; playfulness; qualidade do design do sistema. 


\subsection{Proposta de dimensões da qualidade do Site}

De acordo com o estudo de Zhang e Dran (2002) que indicou que um site deve apresentar determinados atributos, porém, estes não são suficientes para provocar uma perceção positiva ou evitar perceções negativas da qualidade do site, enquanto que, outras características, não sendo necessárias, aumentam a perceção positiva da qualidade do site. Como resultado do estudo os autores compilaram uma extensa lista de atributos agrupadas em onze dimensões que se apresentam no quadro I:

\section{Quadro I}

Listagem das dimensões da qualidade propostas para um site

\begin{tabular}{ccc}
\hline Conteúdo de informação & User empowerment & Organização da informação \\
\hline Resultados cognitivos & Aparência visual & Credibilidade \\
\hline Prazer & Suporte técnico & Imparcialidade \\
\hline Privacidade & Navegação & \\
\hline
\end{tabular}

Fonte: (Zhang e Dran, 2002)

\section{DISCUSSÃO DOS MODELOS DE AVALIAÇÃO DE WEBSITES}

Para Rababah et al. (2011), e um vasto conjunto de académicos, a qualidade, o web-design e a usabilidade são os três principais tipos de avaliações que devem ser realizados aos websites.

1. A Qualidade do site pode ser avaliada pelo Modelo de Kano para a Qualidade. Este modelo avalia o produto e o serviço em três níveis:

- Esperado: corresponde ao mínimo de atributos e propriedades necessários para o funcionamento do site;

- Normal: são as necessidades do cliente e os atributos e propriedades do site que satisfazem, ou não, os consumidores;

-Emocionante: é a perceção de qualidade mais elevada, correspondendo aos atributos e propriedades que surpreendem, muito positivamente, os clientes.

De acordo com Rababah et al. (2011) um website deve tentar suprir as necessidades dos clientes de modo a garantir visitas e conquistar a sua lealdade.

Os fatores de qualidade na web parecem ter um impacto sobre a aceitação do utilizador, pois esses fatores influenciam as crenças e intenção comportamental do utilizador, além disso, a empresa pode ter controle sobre esses fatores (Hernández et al., 2009). 


\section{Tomé Rodrigues e António Andrade}

A qualidade do website afeta o posicionamento que os utilizadores têm da empresa, pois é a forma através da qual as operações são conduzidas (Hernández et al., 2009).

2. O Web-design do site pode ser avaliado pelo Interface Objetos/Ações que se foca na relação que se observa no site entre o interface e a tarefa. Este modelo indica cinco categorias principais, que devem ser consideradas quando se desenha um site: o carregamento da página, o conteúdo, a eficiência na navegação, a segurança e a comercialização. Para Rababah et al. (2011) o carregamento da página é o fator mais importante no design do site.

De acordo com os autores Leuthold et al. (2011) os websites necessitam de sistemas de navegação rápidos e eficazes. Para chegar a esses sistemas de navegação, é necessário distinguir entre duas tarefas sequenciais, por exemplo: A tarefa de organizar os itens em grupos significativos; e a tarefa de design de navegação, lidando com a questão de como exibir os links e os mecanismos de navegação, a fim de tornar a navegação do utilizador eficiente e eficaz.

3. A Usabilidade, desempenha um papel fundamental no processo de design human-centered, uma vez que facilita a conceção de um website de acordo com princípios de engenharia de usabilidade. Como a usabilidade é definida como uma relação entre o utilizador, tarefas, e o propósito do sistema, não existe uma definição simples ou uma medida única de usabilidade (Granic et al., 2010).

A usabilidade está relacionada a facilidade de uso e facilidade de aprendizagem e muitas vezes, só pode ser avaliada com base na experimentação, pelo que todos os projetos, incluindo design e desenvolvimento do website, devem ser submetidos a testes de usabilidade e métodos de validação (Granic et al., 2010; Rababah et al., 2011). Para os mesmos autores as páginas Web devem, ainda, ser concebidas para fácil utilização e entendimento. No entanto, os autores advertem que um site com boa usabilidade não garante a preferência dos consumidores.

Os autores Lin e $\mathrm{Lu}$ (2000) testaram o efeito da qualidade do site sobre as perceções relativas à usabilidade e utilidade. Para os autores a avaliação é interdependente entre três fatores: qualidade da informação; tempo de resposta e acessibilidade. 
Os autores Carvalho et al. (2005), apresentam um esquema representado na figura I que propõe um referencial tridimensional para auxiliar o utilizador na análise da informação de um site.

\section{Figura I}

Referencial para avaliação da autoria e informação de um site

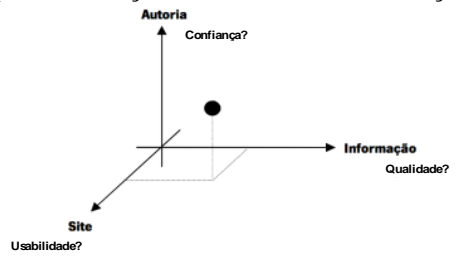

Fonte: (Carvalho et al., 2005)

\subsection{Modelos de avaliação da qualidade}

Um dos primeiros modelos de avaliação da qualidade de um site identificava: pano de fundo; dimensão das imagens; exibição de arquivos de som e endosso de celebridades como fatores importantes de qualidade. A estes fatores rapidamente se adicionaram fatores externos, tais como: serviços gerais, serviços de atendimento específico, e experiência emocional (Dreze e Zufryden, 1997).

$\mathrm{O}$ e-satisfaction model foi o segundo grande modelo de avaliação da qualidade de websites característico por cinco fatores: conveniência; oferta de produtos; informações sobre produtos; design do site; e segurança financeira (Rababah et al., 2011).

O modelo de qualidade do site ou WebQual foi desenvolvido para portais de escolas de gestão, baseado em fatores de facilidade de uso, experiência, informação e comunicação, e integração. Este modelo consiste numa adaptação do modelo SERVQUAL. A segunda geração WebQual 2,0 mede fatores de qualidade como tangibilidade, confiabilidade, recetividade, segurança e empatia (Barnes e Vidgen, 2002). 


\section{Quadro II}

Síntese de critérios de avaliação da qualidade de um website

\begin{tabular}{|c|c|c|c|}
\hline Autor & Perspectiva & Forças & Fraquezas \\
\hline $\begin{array}{l}\text { (Schubert \& Dettling, } \\
2002)\end{array}$ & $\begin{array}{l}\text { Ferramenta } \\
\text { desenvolvida } \\
\text { especificamente para } \\
\text { avaliação de sites de e- } \\
\text { commerce }\end{array}$ & $\begin{array}{l}\text { Enfase na perspectiva } \\
\text { do cliente }\end{array}$ & $\begin{array}{lr}\text { Concentra-se } & \text { em } \\
\text { questőes genéricas } \\
\text { web com pouca } \\
\text { consideração de de } \\
\text { questőes importantes } \\
\text { para a venda }\end{array}$ \\
\hline $\begin{array}{l}\text { (Bames \& Vidgen, } \\
\text { 2002) }\end{array}$ & 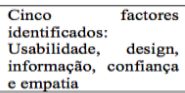 & $\begin{array}{l}\text { Baseado na percepção } \\
\text { do cliente da qualidade } \\
\text { ponderada } \\
\text { importância }\end{array}$ & Foco muito estreito \\
\hline $\begin{array}{l}\text { (Akhter, Hobbs, } \\
\text { Maamar, 2005) }\end{array}$ & $\begin{array}{l}\text { Compara a confiança } \\
\text { dos clientes com a sua } \\
\text { familiaridade com o } \\
\text { local, e medidas } \\
\text { objectivas } \\
\text { segurança. }\end{array}$ & $\begin{array}{l}\text { Identifiea como essas } \\
\text { são importantes no no } \\
\text { incentivo à confiança }\end{array}$ & $\begin{array}{l}\text { Destinado } \\
\text { exclusivamente para } \\
\text { avaliar a confiança e } \\
\text { não de outros } \\
\text { factores importantes } \\
\text { para a venda }\end{array}$ \\
\hline (Lightner, 2004) & $\begin{array}{l}\text { Avaliação de uma } \\
\text { perspectiva } \\
\text { atendimento ao eliente }\end{array}$ & $\begin{array}{l}\text { Estudo da concepção } \\
\text { está subordinado às } \\
\text { funçoóes efectivamente } \\
\text { prestadas }\end{array}$ & $\begin{array}{l}\text { Năo cobre todo } \\
\text { ciclo de venda }\end{array}$ \\
\hline $\begin{array}{l}\text { (Hahn, Kauffman, \& } \\
\text { Park, 2002) }\end{array}$ & $\begin{array}{lr}\begin{array}{l}\text { Avaliação } \\
\text { perspectiva } \\
\text { investimento }\end{array} & \begin{array}{r}\text { numa } \\
\text { de }\end{array} \\
\end{array}$ & $\begin{array}{l}\text { O foco } \\
\text { administração }\end{array}$ & $\begin{array}{l}\text { Identifica áreas com } \\
\text { problemas, mas não } \\
\text { dá soluçōes }\end{array}$ \\
\hline
\end{tabular}

Fonte: (Rababah et al., 2011)

Contudo, Rababah et al. (2011) chegaram à conclusão de que não existe uma abordagem totalmente integrada, pelo que, inferem que há necessidade de uma abordagem global que combine todos os fatores que afetam a qualidade (ver quadro II).

\subsection{Modelo de avaliação da qualidade de um portal}

Os autores Yang et al. (2005) basearam o seu modelo de avaliação em seis atributos de qualidade do serviço de um portal. Estes podem constituir um quadro funcional preliminar para uma avaliação mais aprofundada da qualidade do serviço do portal. Estes atributos, também, contribuem para a validade de conteúdo a ser medido (ver figura II).

\section{Figura II}

Modelo de avaliação da qualidade do serviço de um portal

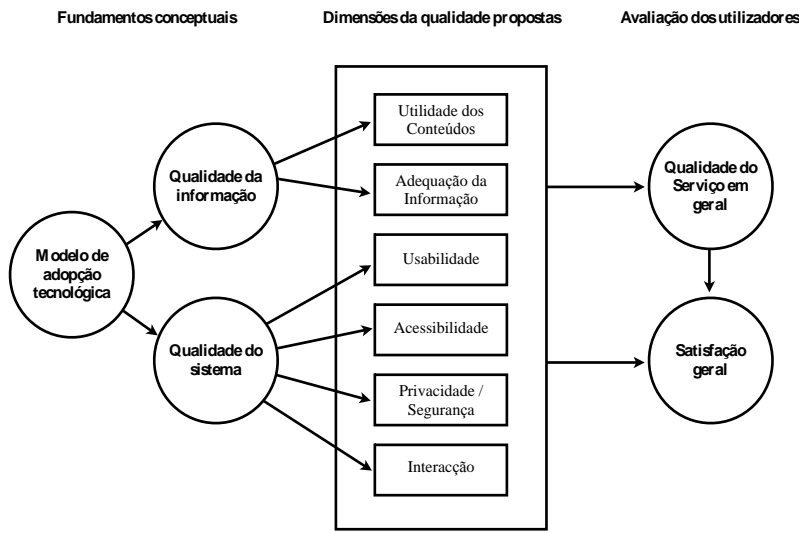

Fonte: (Yang et al., 2005) 


\section{Adoção do portal como meio de informação e comunicação}

Os autores Yang et al. (2005) sugerem que a decisão dos utilizadores de adotar uma tecnologia de informação é principalmente determinada pela sua atitude em relação à utilidade e facilidade de utilização. Se a utilidade e facilidade de uso da informação e comunicação através da Internet não compensarem possíveis perdas para os utilizadores causadas por experiências impessoais, dificuldades técnicas, e esforço de aprendizagem, entre outras, os utilizadores podem simplesmente voltar aos canais tradicionais. Assim, para Yang et al. (2005) a questão a responder é: quais os aspetos de utilidade e facilidade de uso de portais Web que os clientes esperam?

\section{Qualidade da informação}

Corresponde à perceção dos utilizadores sobre a qualidade da informação apresentada num site. Os determinantes do sucesso do sistema de informação são a importância da relevância, exatidão e atualidade da informação (Yang et al., 2005).

As dimensões da qualidade da informação propostas são as seguintes:

- Utilidade do conteúdo. Refere-se ao valor, à confiabilidade e precisão das informações. Especificamente, o valor da informação está relacionado com a relevância e clareza. Confiabilidade das informações refere-se à sua exatidão, confiabilidade e consistência. Precisão da informação está ligada à atualidade da informação e atualização contínua (Yang et al., 2005).

- Adequação da informação. Refere-se ao grau de integridade das informações. Os portais necessitam de fornecer informações para facilitar a compreensão do utilizador sobre produtos e sistemas para apoiar a tomada de decisão (por exemplo, descrição detalhada do produto, informações transparentes de preços). No entanto, os utilizadores precisam de serviços complementares, tais como informações sobre a empresa, conselhos profissionais, relatórios de pesquisas, links para sites relevantes, informações de contacto, e arquivos (Yang et al., 2005).

\section{Qualidade do sistema}

Refere-se à perceção que os utilizadores têm do desempenho de um portal na recuperação da informação e entrega. Para medir a qualidade do sistema, a facilidade de utilização tem sido considerada como o fator mais frequentemente utilizado na medição do sucesso ou satisfação do utilizador. No contexto do portal, a qualidade do sistema depende de 
fatores como a interatividade, navegação, acesso, hiperlinks, entretenimento, segurança e privacidade (Yang et al., 2005). Estes fatores podem ser englobados nos quatro atributos seguintes:

- Usabilidade. Identificam-se vários fatores, principalmente de layout de conteúdo e classificação, estrutura do site, interface do utilizador, aparência e design visual, intuição, capacidade de leitura/ compreensão/ clareza, facilidade de pesquisa, e facilidade de navegação. Um requisito fundamental é a adequação técnica, no que diz respeito a recursos técnicos do portal, por exemplo, capacidades dos sistemas, a integridade do hardware de rede e software do sistema (Yang et al., 2005).

- Acessibilidade. O benefício conveniência de usar um portal como um centro de informação não pode ser alcançado sem acessibilidade. Trata-se fundamentalmente de dois aspetos: disponibilidade e tolerância. Os utilizadores esperam que os serviços on-line estejam disponíveis 24 horas todos os dias e, também, rapidez de download da página, de acesso e pesquisa (Yang et al., 2005).

- Privacidade e segurança. Os portais coletam frequentemente uma variedade de informações pessoais dos seus utilizadores. Assim, os recursos de privacidade e segurança tornaram-se uma preocupação séria. Algumas medidas frequentemente utilizadas incluem garantias de proteção da informação pessoal dos utilizadores. A confiança e a reputação da organização são fatores a considerar para a avaliação (Yang et al., 2005).

- Interação. Envolve três tipos de operações entre: utilizadores e funcionários prestadores de serviços, utilizadores e o portal, e entre utilizadores de produtos similares. Embora o uso de um portal seja essencialmente um processo de uso autónomo, os utilizadores podem esperar receber serviços personalizados ou customizados a partir de uma pessoa de contacto. Os serviços, também podem ser prestados automaticamente, sem intervenção humana ou usando email, fóruns, salas de chat, e fórum de discussão (Yang et al., 2005).

Segundo Yang et al. (2005) cada um dos seis fatores identificados aos níveis da qualidade de informação e qualidade do sistema têm um impacto significativo na qualidade geral do serviço. Através da compreensão das dimensões de qualidade de serviço para portais, uma organização terá uma hipótese muito maior de ganhar mais negócios e servir melhor os interesses dos seus stakeholders. Para os gestores, os seis 
fatores podem servir um modelo útil de diagnóstico. Os gestores podem utilizar o modelo para medir e melhorar o serviço.

\subsection{MODELO WDP}

Os autores Conte et al. (2009) aplicaram o modelo $\mathrm{WDP}^{\S}$ para avaliar o web-design e usabilidade. Este modelo identifica três perspetivas de design maioritariamente utilizadas no desenvolvimento da Web:

- Conceptual: representa os elementos conceptuais que compõem o domínio da aplicação;

- Apresentação: representa as características relacionadas com a aplicação ao nível de layout e disposição dos elementos do interface;

- Navegação: representa o espaço de navegação, definindo os elementos de acesso à informação e às suas associações.

No estudo que realizaram os autores Conte et al. (2009) fizeram uma comparação entre a avaliação Heurística e o modelo WDP, constatando que o modelo WDP obtém melhor performance, independentemente do nível técnico dos avaliadores.

O modelo WDP é versátil, uma vez que permite uma visão sobre o design e sobre a usabilidade de forma integrada. Apresenta-se a síntese do modelo no quadro seguinte:

\section{Quadro III}

Relação entre as perspetivas de web-design e avaliação de usabilidade

\begin{tabular}{ll}
\hline Perspectiva de Web-design & Foco de Usabilidade \\
\hline & $\begin{array}{l}\text { Relaciona-se com a clareza e a concisão de problemas nos } \\
\text { elementos do domínio. Nesta perspectiva a usabilidade é }\end{array}$ \\
satisfatório se diferentes utilizadores facilmente \\
compreenderem os termos do domínio, o que evita erros \\
causados por termos ambíguos, inconsistentes ou \\
desconhecidos. \\
\hline Quão coerente é a informação apresentada ao utilizador? Nesta \\
perspectiva a usabilidade é satisfatória se a disposição dos \\
elementos do interface permitem ao utilizador realizar tarefas \\
de forma eficaz, eficiente e agradável. \\
\hline Diz respeito ao acesso de diferentes utilizadores às \\
funcionalidades do sistema. Nesta perspectiva a usabilidade é \\
satisfatória se as opções de navegação permitirem ao utilizador \\
realizar as suas tarefas de forma eficaz, eficiente e agradável.
\end{tabular}

Fonte: (Conte et al., 2009)

\footnotetext{
$\S$ Web Design Prespectives-based Usability Evaluation
} 


\subsection{Modelo de avaliação de modelagem de web-design}

O modelo proposto por Yen e Wang (2007) consiste numa avaliação realizada em três camadas:

\section{- Application layer;}

- Generic website design layer;

- Graph modeling layer.

Deste modo é possível fazer em simultâneo uma avaliação, modelagem e aperfeiçoamento do web-design de um site.

A figura seguinte ilustra os componentes do modelo de avaliação e modelagem de web-design de acordo com Yen e Wang (2007):

Figura III

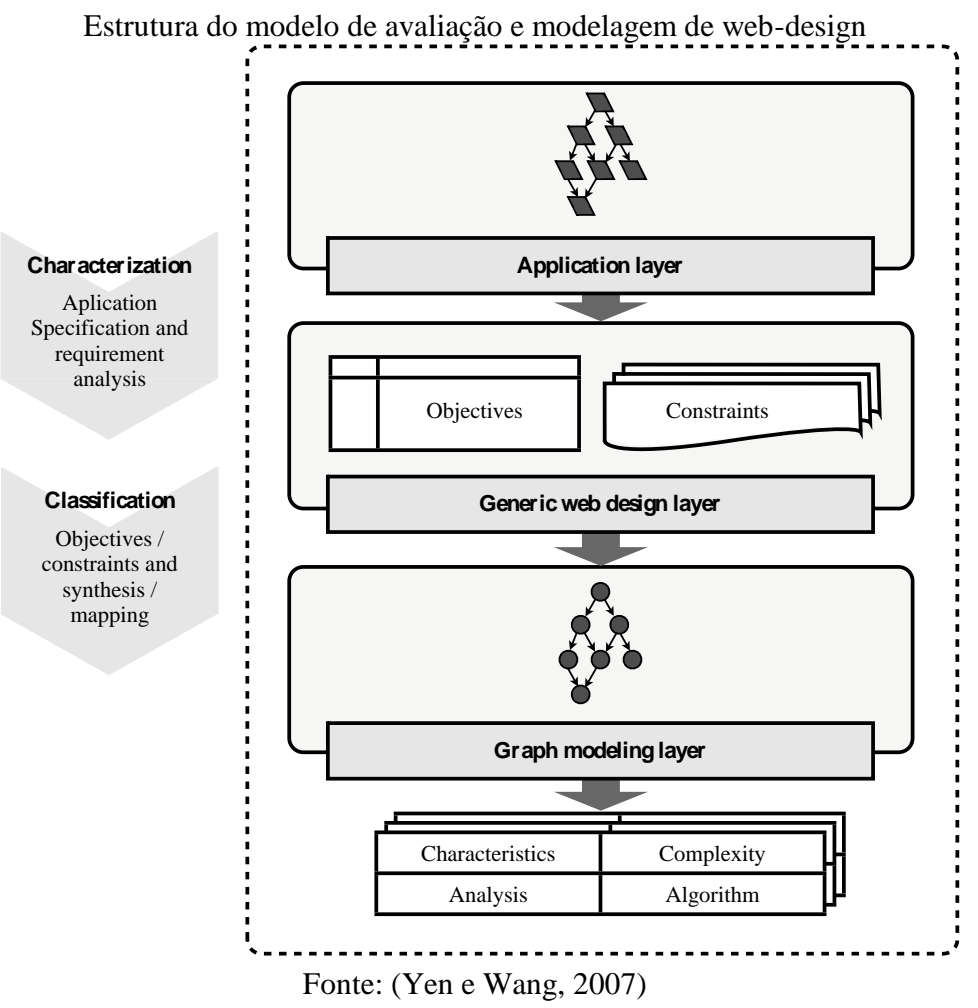

Segundo Yen e Wang (2007) as organizações criam sites para fins diferentes, que vão desde a divulgação de informações ou para suportar transações online. Os sites variam consideravelmente em requisitos. Por exemplo, sites típicos no setor financeiro têm rigorosos requisitos de 
cache em comparação com os utilizados no setor da educação. Sites executados por organizações do mesmo setor, também, diferem nos requisitos que exigem em geral, design específico, estrutura, layout de página, e de apoio à navegação.

O objetivo principal de um site pode ser a promoção de produtos, prestação de serviços, processamento de transações ou de serviço ao cliente (Yen e Wang, 2007).

- A application layer serve para realizar as descrições de design do site ou de avaliação tendo por base a indústria (por exemplo, finanças ou entretenimento), a finalidade primária (por exemplo, informativo ou de transação), e requisitos de desempenho essenciais (por exemplo, tempo de carregamento de página, o número médio de cliques para aceder ao conteúdo). Um cenário de design do site descrito na application layer pode ser transformado para a generic website design layer criando, assim, uma instância equivalente na camada do meio (Yen e Wang, 2007).

- Na generic website design layer, define-se um conjunto de categorias de conceção genérica que diferem nos seus objetivos e restrições. $\mathrm{O}$ objetivo de um site pode ser analisado a partir da perspetiva do utilizador ou do designer considerando-se ambas as perspetivas para obter o melhor output. Os utilizadores valorizam a pesquisa de informação eficaz e eficiente e facilidade de acesso ao site. Para e-commerce, os visitantes preferem aceder a informações pertinentes do produto, rapidamente ou com menos cliques. Os consumidores, também, dão valor a comparações de produtos para garantirem a melhor tomada de decisão de compra (Yen e Wang, 2007).

A fronteira entre objetivo do projeto e as restrições pode ser ambígua. Em geral, o objetivo representa a motivação ou o objetivo principal de um site, enquanto as restrições descrevem os requisitos que devem ser satisfeitos para alcançar o objetivo (Yen e Wang, 2007).

- Na graph modeling layer dá-se a modelagem do projeto genérico como um problema de configuração gráfico de construção, que permite a busca de um projeto ideal e, ao mesmo tempo, suporta avaliações analíticas do design do site (Yen e Wang, 2007). Deste modo, utiliza-se o objetivo principal e as restrições chave para definir uma categoria de design genérico de site e modelá-lo como um problema gráfico particular. 
Segundo Yen e Wang (2007) notações matemáticas do projeto genérico são necessárias para facilitar a análise analítica do design do site.

\section{Quadro IV}

Características importantes relacionadas com o conteúdo no design do site

\begin{tabular}{ll}
\hline Dimensão & Descrição \\
\hline Precisão & $\begin{array}{l}\text { A precisão das informações e a capacidade de leitura deve ser } \\
\text { aceitável }\end{array}$ \\
\hline Relevância e abrangência & $\begin{array}{l}\text { A relevância e abrangência de informações fornece informações } \\
\text { relevantes para ajudar o utilizador a navegar e a comprar on-line }\end{array}$ \\
\hline Formato de média & Formato de média é a forma como a informação é apresentada no site \\
\hline Oportunidade & A actualidade da informação no site \\
\hline
\end{tabular}

Fonte: (Yen e Wang, 2007)

No seu estudo Yen e Wang (2007) propuseram uma série de características importantes para o conteúdo do design da página web como se pode verificar pelo quadro IV e características a considerar no design como se pode constatar pelo quadro $\mathrm{V}$.

\section{Quadro V}

Sumário de características orientadas para o design do site

\begin{tabular}{ll}
\hline Dimensão & Descrição \\
\hline Tamanho & O número de páginas do site \\
\hline Layout da página & O formato de uma única página da Web \\
\hline Estrutura / navegação & $\begin{array}{l}\text { Existem estruturas de árvore e gráficas. Estruturas diferentes } \\
\text { fornecem diferentes tipos de recursos de acesso ou navegação }\end{array}$ \\
\hline Tempo de resposta & $\begin{array}{l}\text { A velocidade de acesso e disponibilidade do site em todos os tempos. } \\
\text { O tempo de procura e o número de cliques para aceder à página de } \\
\text { destino deve ser o mais curto possível }\end{array}$ \\
\hline Segurança & $\begin{array}{l}\text { A capacidade do site para proteger informações pessoais do } \\
\text { consumidor coletadas a partir das suas transações electrónicas }\end{array}$ \\
\hline & Fonte: (Yen e Wang, 2007)
\end{tabular}

\subsection{Modelo de avaliação da usabilidade}

Os autores Llanos e Muñoz (2007) e Yusof et al. (2010) indicaram que existem muitas abordagens possíveis para a avaliação de usabilidade de um site. Alguns dos métodos mais comuns são:

1. Inspeção de usabilidade: nesta abordagem, um grupo de especialistas em usabilidade são convidados a julgar e comentar o design do interface do utilizador em relação aos serviços prestados; 
2. Realização de teste de utilizador final: um grupo de utilizadores (target) são convidados a testar o site. O desempenho dos utilizadores que completam a tarefa formal é registado e analisado;

3. Checklist de usabilidade por peritos de usabilidade: é um teste efetuado por técnicos com formação para avaliar a usabilidade de um site;

4. Testes de audição: o utilizador final ou o público-alvo é orientado por um aplicativo de teste através do site. Todos os comentários e as reações dos utilizadores são gravados para serem analisadas (Yusof et al., 2010).

De acordo com o estudo de Yusof et al. (2010), alguns autores sugerem, também, a utilização de inspeções de usabilidade por ser um método menos dispendioso. No entanto, Llanos e Muñoz (2007) indicam que este método da avaliação da usabilidade é muito subjetivo. No método de inspeções de usabilidade, o resultado da avaliação depende muito das competências dos inspetores e da sua experiência. Além disso, são produzidos resultados diferentes quando avaliações ao mesmo site são feitas por inspetores diferentes.

O envolvimento dos utilizadores na avaliação da usabilidade é muito importante (Yusof et al., 2010), uma vez que, problemas funcionais são descobertos através da observação e interação com o utilizador, enquanto estes executam determinadas tarefas. Deste modo, a opinião dos utilizadores auxilia o projetista do site na escolha do layout mais adequado (Llanos e Muñoz, 2007).

No quadro VI estão explanados os critérios propostos por Llanos e Muñoz (2007) e respetivos indicadores.

Quadro VI

Critérios de medição da usabilidade de um site

\begin{tabular}{ll}
\hline Critério de usabilidade & Indicadores de medição \\
\hline Aprendizagem & $\begin{array}{l}\text { Tempo médio que o utilizador despende para realizar uma tarefa } \\
\text { especifica }\end{array}$ \\
\hline Satisfação do utilizador & $\begin{array}{l}\text { Inquirir o utilizador após a utilização do site, para medir o seu grau } \\
\text { de satisfação }\end{array}$ \\
\hline Confiabilidade & $\begin{array}{l}\text { Capacidade do utilizador cumprir de modo correcto uma tarefa } \\
\text { específica }\end{array}$ \\
\hline Eficiência & $\begin{array}{l}\text { Forma como os utilizadores usam o sistema para completar uma } \\
\text { determinada tarefa }\end{array}$ \\
\hline
\end{tabular}

Fonte: (Llanos e Muñoz, 2007)

Os autores Granic et al. (2010) chegaram à conclusão que se deve fazer uso de várias técnicas de avaliação de usabilidade com vista a obter 
uma avaliação mais abrangente, uma vez que, a usabilidade de um portal é mais do que a usabilidade e design das suas partes, pois um portal também tem de cuidar de questões mais gerais ao nível da "embalagem" das suas ofertas, nomeadamente: estruturação; integração e organização. Outra questão fundamental é a adaptação que um portal deve apresentar para um grupo de utilizadores específicos ou para um grupo de utilizadores mais vasto (Granic et al., 2010).

Um estudo feito por Shelat e Stewart (2004) já advertia para o facto da necessidade de uma avaliação da usabilidade a vários níveis. Nesse estudo foram realizados testes a um portal de viagens em que os participantes utilizadores finais realizaram testes com doze tarefas. Os testes incluíam o método de gravação do que os participantes diziam durante o teste, questionário, entrevista e sessão de esclarecimento.

\subsection{Modelo de avaliação estratégico}

Num estudo realizado pelos autores Chiou et al. (2010) sobre os vários modelos de avaliação e conceção de websites, foi possível a elaboração de um modelo genérico que visa identificar os elementos críticos para a conceção e desenvolvimento do comércio on-line, abordando, simultaneamente, os aspetos estratégicos, operacionais, organizacionais e questões técnicas. Na prática, cada empresa tem as suas próprias estratégias, que devem ser consideradas no desenvolvimento de um quadro de avaliação. Nesse sentido os autores sugerem que esta se faça de acordo com o Marketing Mix.

Este modelo é desenvolvido em cinco passos como se pode verificar pela figura seguinte: 
Figura IV

Estrutura do modelo de avaliação estratégico

\begin{tabular}{|c|}
\hline Primeira etapa - Entrevista ao gestor web \\
\hline $\begin{array}{l}\text { 1. Identificar a estratégia web } \\
\text { 2. Determinar critérios e ponderações }\end{array}$ \\
\hline Segunda etapa - Desenvolvimento \\
\hline $\begin{array}{l}\text { 1. Listar objectivos e acções do website } \\
\text { 2. Desenvolver questionários }\end{array}$ \\
\hline Terceira etapa - Avaliação do website \\
\hline $\begin{array}{l}\text { Conduzir avaliaç̃es do website com base } \\
\text { num painel de peritos, utilizando linguagem } \\
\text { fuzzy }\end{array}$ \\
\hline Quarta etapa - Pesos e pontuações \\
\hline $\begin{array}{l}\text { 1. Transformar linguagem fuzzy em números } \\
\text { 2. Normalizar critérios e ponderações } \\
\text { 3. Calcular pesos e pontuações }\end{array}$ \\
\hline Quinta etapa - Análise dos dados \\
\hline $\begin{array}{l}\text { 1. Listar pesos, pontuações e gaps por critério } \\
\text { 2. Elaborar matriz de performance de critério } \\
\text { 3. Elaborar gráfico radar para } 4 \mathrm{PsC}\end{array}$ \\
\hline
\end{tabular}

Fonte: (Chiou et al., 2010)

1. Na primeira fase deve ser realizada uma entrevista com o gestor do site para ser identificada a estratégia do website (metas, objetivos e ações). Após a entrevista devem ser selecionados os critérios de avaliação que se enquadrem na estratégia. Em seguida deve ser construída uma estrutura hierárquica para delinear a relação entre as metas, objetivos, ações e critérios. O peso de cada critério deve ser definido pelo gestor do site (Chiou et al., 2010).

2. Na segunda fase, desenvolve-se um instrumento de avaliação webbased com duas secções principais:

- Secção 1 contém o resumo da entrevista com o gestor do site, para ser referência para os avaliadores;

- Secção 2 enumera os questionários criados com base nos critérios selecionados (Chiou et al., 2010).

3. Na terceira fase, procede-se a uma avaliação do website com a ajuda de especialistas, utilizando termos linguísticos fuzzy para expressar a sua concordância ou discordância com o que se perguntou em cada questionário (Chiou et al., 2010). 
4. A quarta fase, corresponde ao momento em que se calculam os pesos e critérios de pontuação. Esta fase é subdividida em três etapas:

- Etapa 1: transformar termos linguísticos em números.

- Etapa 2: normalizar os pesos dos critérios para comparar a importância relativa de cada um.

- Etapa 3: calcular a pontuação ponderada dos objetivos e critérios (Chiou et al., 2010).

5. Na quinta fase, realiza-se e discute-se a análise dos dados, a partir de três perspetivas.

- Primeiro: o peso e importância de cada critério são indicados para comparar pontos fortes e fracos. Em geral, os critérios com pontuação mais baixa devem ser melhorados. No entanto, há que ter em consideração a relevância do critério, para não se correr o risco de tentar melhorar algo que não é importante. A questão de quão bem o website satisfaz os seus objetivos estratégicos obtém-se através da pontuação ponderada dos objetivos.

- Segundo: uma matriz de critérios de desempenho deve ser construída para fornecer aos gestores as informações de desempenho e para classificar as prioridades do plano de melhoria.

- Terceiro: os critérios são classificados nas dimensões $4 \mathrm{PsC}^{* *}$ seguindo os critérios de classificação anteriores. Posteriormente, os pesos de cada critério dimensional e pontuação são adicionados para obter o peso médio e a pontuação das dimensões dos 4 PsC. Um gráfico de radar pode ser construído para identificar os pontos fortes e fracos das dimensões dos 4PsC. Ao comparar o peso de cada dimensão e pontuação, pode-se determinar uma dimensão que é importante, mas que pode ter uma importância relativa. Uma análise drill-down pode ser usada para ajudar os gestores a descobrir os critérios que têm pontuações relativamente baixas (Chiou et al., 2010).

** Marketing Mix (Product, Price, Promotion, Place and Customer Relationship) 


\section{PROPOSTA DE MODELO DE AVALIAÇÃo A 4 DIMENSÕES}

Em primeiro lugar, importa referir que o modelo proposto servirá como ferramenta de apoio à conceção do website e à sua avaliação à priori. No entanto, permitirá que à posteriori sejam feitas avaliações com o objetivo de evidenciar as dimensões que poderão vir a ser melhoradas.

\section{Figura V}

Modelo de avaliação de websites a 4 dimensões

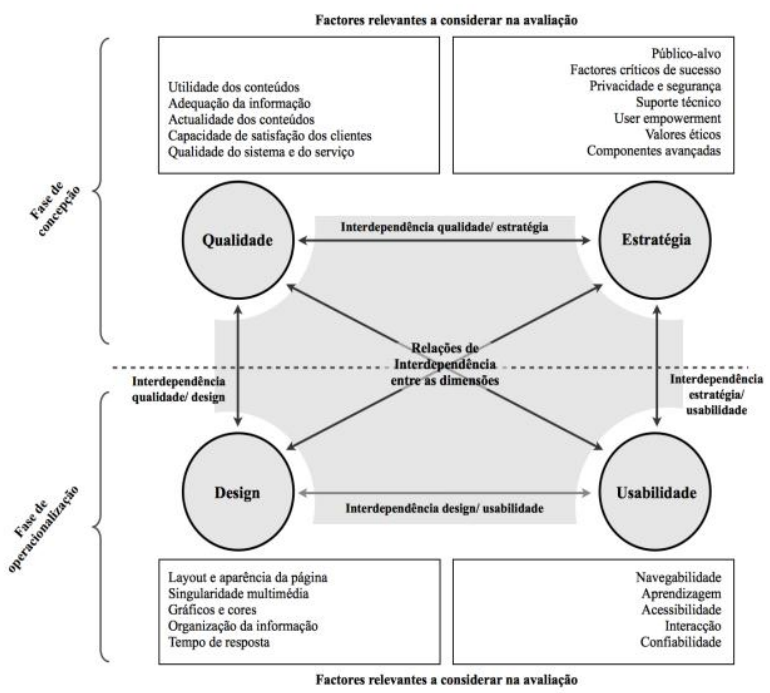

Fonte: Dados próprios

Os fatores relevantes a serem considerados para cada uma das dimensões poderão ser alterados de acordo com as necessidades que sejam identificadas para cada caso específico.

O modelo prevê duas fases de desenvolvimento:

- Conceção: nesta fase devem ser avaliadas as dimensões qualidade e estratégia, quais as melhores opções a tomar para satisfazer os utilizadores e como se pretende posicionar o website. Como consequência da interdependência entre as dimensões nesta fase fica definido quais os objetivos de web-design e usabilidade. As medidas tomadas nesta fase vão dar origem à fase de operacionalização. 
- Operacionalização: após terem sido determinados os objetivos na fase anterior avalia-se o webdesign e a usabilidade de modo a garantir congruência com as outras dimensões.

Deste modo, em cada uma das dimensões do modelo será feita a análise, síntese, avaliação e tomada a decisão, perante os dados obtidos, como se propõe na figura seguinte:

Figura VI

Modelo processual de tomada de decisão por dimensão do modelo

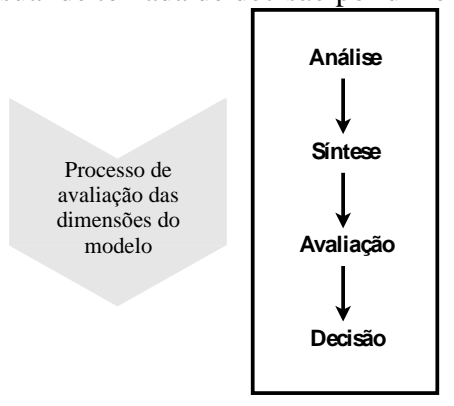

Fonte: Dados próprios

- Análise: deve ser realizada por forma a detetar quais os critérios mais relevantes na ótica do utilizador/público alvo.

- Síntese: os dados obtidos na análise devem ser expostos na forma de critérios simples e, possíveis formas de os avaliar (indicadores).

- Avaliação: os critérios a avaliar deverão ser realizados a uma amostra que componha programadores e utilizadores.

- Decisão: após os resultados da avaliação o responsável pelo website deve decidir com base nos dados ao seu dispor.

É importante referir que a avaliação deve ter por base a escala proposta no Modelo de Kano (esperado, normal, emocionante), com recurso à metodologia de escala de Likert.

A recolha de dados pode ser baseada em inquéritos por questionário, no entanto, o modelo suporta eventuais ponderações e tratamento matemático.

\section{CONCLUSÕES}

Não se verifica conformidade entre os autores face às dimensões de avaliação de um website e/ou portal. No que se refere aos critérios de avaliação os autores também não são unânimes. 
A maioria das ferramentas desenvolvidas para a avaliação de websites têm por base a análise de rácios e inquéritos. Tal procedimento apresenta resultados significativos, mas exige bastante tempo para a coleta e análise dos dados em cada fase de medição. Deste modo, verifica-se a necessidade de uma metodologia de análise que vise a diminuição de tempo e, possa ser realizada antes da colocação do site na internet para dessa forma trazer mais rigor à avaliação de websites, em particular de ecommerce.

A usabilidade e o web-design são aspetos muito importantes de uma página da Web. Se a usabilidade não for considerada durante a fase do projeto, serão verificados, no futuro, impactos negativos sobre o sucesso do site. Do ponto de vista do negócio, o site perderá oportunidades.

O modelo da avaliação estratégico, muito útil para sites de ecommerce, deve ser posto em prática a par com a conceção do site, com vista a minimizar futuros problemas de qualidade, web-design e usabilidade.

Os autores exprimem, por último, a mesma opinião sobre o sucesso de um website. Segundo estes, o sucesso não depende exclusivamente da sua qualidade, web-design, usabilidade e estratégia, mas sim, de uma boa integração das dimensões de avaliação, com variáveis externas ao site, que se traduzem num acréscimo de valor para o utilizador.

O modelo de avaliação a quatro dimensões proposto pretende minimizar os impactos negativos supra referidos, podendo inclusive tirar partido dos pontos fortes dos modelos abordados.

Por outro lado, o modelo pode ser, ainda, aplicado aos media sociais.

O modelo a quatro dimensões pretende ser uma ferramenta ao dispor do gestor e dos programadores, para que se possam conjugar opções estratégicas com tecnologia, de modo a proporcionar a melhor experiência possível ao utilizador. O modelo tem a particularidade de contrapor testes a utilizadores e programadores, sendo bastante orientado para o utilizador. Pelo que se pode dizer que o modelo potencia a competitividade do website num mundo tão competitivo como é o da internet, pois prevê que sejam feitos testes à priori e à posteriori do site entrar na internet. 


\section{REFERÊNCIAS}

AKHTER, F., HOBBS, D. e MAAMAR, Z. (2005), “A fuzzy logicbased system for assessing the level of business-to-consumer trust in electronic commerce", Expert Systems with Applications, 28, 623628 ;

BAI, B., LAW, R. e WEN, I. (2008), "The impact of website quality on customer satisfaction and purchase intentions: Evidence from Chinese online visitors", International Journal of Hospitality Management, 27 (3), 391-402;

BARNES, S.J. e VIDGEN, R.T. (2002), "An integrative approach to the assessment of E-commerce quality", Journal of Electronic Commerce Research, 3 (3), 114-127;

BELLAS, F. (2004), "Standards for second-generation portals", IEEE Internet Computing, 8, 54-60;

BLANKENSHIP, E. (2001), Portal design vs web design, (3rd ed.). SAP user experience, SAP;

CARVALHO, A.A.A., SIMÕES, A. e SILVA, J.P. (2005), "Indicadores de qualidade e de confiança de um site", Cied Universidade do Minho, 2, 19-31;

CHIOU, W., CHIN-CHAO, L. e PERNG, C. (2010), “A strategic framework for website evaluation based on a review of the literature from 1995-2006", Information \& Management, 47 (5-6), 282-290;

CONTE, T., VAZ, V., MASSOLAR, E. M. e TRAVASSOS, G.H. (2009), "Improving a Web Usability Inspection Technique Using Qualitative and Quantitative Data from an Observational Study", In 2009 XXIII Brazilian Symposium on Software Engineering, Dcc, 227-235;

DREZE, X. e ZUFRYDEN, F. (1997), "Testing web site design and promotional content", Journal of Advertising Research, 37 (2), 77 91;

GRANIC, A., MITROVIC, I. e MARANGUNIC, N. (2010), "Exploring the usability of web portals: A Croatian case study", International Journal of Information Management, 31, 339-349;

HAHN, J., KAUFFMAN, R.J. e PARK, J. (2002), "Designing for ROI: Toward a Value-Driven Discipline for E-Commerce System Design", In Proceedings of the 2002 Hawaii International Conference on System Sciences, Big Island, Hawaii, ACM Press, 2663- 2672; 
HERNÁNDEZ, B., JIMÉNEZ, J. e MARTÍN, M.J. (2009), "Key website factors in e-business strategy", International Journal of Information Management, 29 (5), 362-371;

KIM, S. (2004), "Dimensional hierarchy of retail website quality", Information \& Management, 41 (5), 619-633;

LEUTHOLD, S., SCHMUTZ, P., BARGAS-AVILA, J.A., TUCH, A.N. e OPWIS, K. (2011), "Vertical versus dynamic menus on the world wide web: Eye tracking study measuring the influence of menu design and task complexity on user performance and subjective preference", Computers in Human Behavior, 27 (1), 459472;

LÉVY, P. (2000), “Cibercultura”, Instituto Piaget. Lisboa;

LIGHTNER, N. J. (2004), "Evaluating e-commerce functionality with a focus on customer service", Communications of the ACM, 47 (10), 88-92;

LIN, J.C.C. e LU, H. (2000), "Towards an understanding of the behavioural intention to use a website", International Journal of Information Management, 20, 197-208;

LIU, C. e ARNETT, K.P. (2000), "Exploring the factors associated with website success in the context of electronic commerce", Information \& Management, 38, 23-33;

LLANOS, C.I. e MUÑOZ, M.N. (2007), "Design Guidelines for Web Applications Based on Local Patterns", In Proceedings of the 2007 Euro American conference on Telematics and information systems, ACM New York. USA;

RABABAH, O.M., AL-SHABOUL, M. e Al-SAYYED, R. (2011), "A New Vision for Evaluating the Quality of E-Commerce Websites", International Journal of Advanced Corporate Learning, 4 (1), 32$41 ;$

SCHUBERT, P. e DETTLING, W. (2002), "Extended Web Assessment Method (EWAM) - evaluation of e-commerce applications from the customer's viewpoint", In 35th Hawaii International Conference on Systems Sciences, Hawaii, USA, IEEE 7: 175b;

SHELAT, B. e STEWART, T. (2004), "Usability testing report", Transport direct portal;

SIMÕES, A. (2005), "Análise de Sites para/sobre o Ensino da Matemática e Implicações na Prática Docente”, Braga;

YANG, Z., CAI, S., ZHOU, Z. e ZHOU, N. (2005), "Development and validation of an instrument to measure user perceived service quality 
of information presenting Web portals", Information \& Management, 42 (4), 575-589;

YEN, B., HU, P. e WANG, M. (2007), “Toward an analytical approach for effective Web site design: A framework for modeling, evaluation and enhancement", Electronic Commerce Research and Applications, 6 (2), 159-170;

YUSOF, U.K., KHAW, L.K., YANG, H. e NEOW, B.J. (2010), "Balancing between Usability and Aesthetics of Web Design", In 2010 International Symposium in Information Technology, Kuala Lumpur.

ZHANG, P. e DRAN, G.M. (2002), "User expectations and rankings of quality factors in different website domains", International Journal of Electronic Commerce, 6 (2), 9-33. 